\title{
Palladium-Catalyzed Asymmetric Synthesis of Axially Chiral (Allenylmethyl)silanes and Chirality Transfer to Stereogenic Carbon Centers in $\mathbf{S}_{\mathbf{E}}{ }^{\prime}$ Reactions
}

\author{
Masamichi Ogasawara, ${ }^{*}$ Kazuhito Ueyama, Takashi Nagano, Yoshiyuki Mizuhata, and Tamio \\ Hayashi* \\ Department of Chemistry, Graduate School of Science, Kyoto University, Sakyo, Kyoto 606-8502, \\ Japan
}

\section{Supporting Information}

\section{Experimental Section}

General. All anaerobic and/or moisture sensitive manipulations were carried out with standard Schlenk techniques under predried nitrogen or with glovebox techniques under prepurified argon. NMR spectra were recorded on a JEOL JNM LA500 spectrometer $\left({ }^{1} \mathrm{H}, 500 \mathrm{MHz} ;{ }^{13} \mathrm{C}, 125\right.$ $\mathrm{MHz}) .{ }^{1} \mathrm{H}$ and ${ }^{13} \mathrm{C}$ chemical shifts are reported in ppm downfield of internal tetramethylsilane. Optical rotations were measured on a JASCO DIP-370 polarimeter. (3-Bromopenta-2,4dienyl)trimethylsilane (1), ${ }^{1}[\mathrm{PdCl}(\pi \text {-allyl })]_{2},{ }^{2} \mathrm{dpbp},{ }^{3}{ }^{t} \mathrm{BuCH}(\mathrm{OMe})_{2}(\mathbf{4 z}),{ }^{4} \mathrm{Pd}(\mathrm{dba})_{2},{ }^{5}$ and $(R)-$ segphos 6 were prepared according to the reported methods. All the other chemicals were obtained from commercial sources.

Palladium-Catalyzed Synthesis of (Allenylmethyl)silanes (3a-c). Nonasymmetric synthesis of the (allenylmethyl)silanes 3a-c was conducted according to a reported procedure. ${ }^{7}$ The reaction conditions and results are described in Table 1. A typical procedure is given for the preparation of dimethyl 2-methyl-2-[5-(trimethylsilyl)penta-2,3-dienyl]propane-1,3dioate (3a): A mixture of $\left[\mathrm{PdCl}(\pi \text {-allyl) }]_{2}(1.6 \mathrm{mg}, 8.7 \mu \mathrm{mol} / \mathrm{Pd})\right.$, dpbp $(4.9 \mathrm{mg}, 9.4 \mu \mathrm{mol})$, and $\mathbf{1}$ (104 mg, $474 \mu \mathrm{mol})$ was dissolved in THF $(5 \mathrm{~mL})$ and the solution was added to $\mathrm{Na}\left[\mathrm{CMe}(\mathrm{COOMe})_{2}\right](\mathbf{2 a}, 80.5 \mathrm{mg}, 479 \mu \mathrm{mol})$ by cannula transfer under nitrogen. The mixture was stirred at $23{ }^{\circ} \mathrm{C}$ for $12 \mathrm{~h}$, then filtered through a short pad of $\mathrm{SiO}_{2}$ to remove precipitated inorganic salts. The silica gel pad was washed with small amount of $\mathrm{Et}_{2} \mathrm{O}$ three times and the combined solution was evaporated to dryness under reduced pressure. The yellow residue was chromatographed on silica gel (hexane/Et $2 \mathrm{O}=4 / 1$ ) to give the (allenylmethyl)silane $(122 \mathrm{mg}, 91 \%$ yield; 3a) as colorless oil. The characterization data of the allenic products are listed below.

Asymmetric synthesis of the (allenylmethyl)silanes $(R)-\mathbf{3 a}$ and $(R)-\mathbf{3 b}$ was performed according to a reported method ${ }^{8}$ with slight modifications. The reaction conditions and the results are described in Table 1. A typical procedure is given for the preparation of $(R)$-3a: A mixture of $\mathrm{Pd}(\mathrm{dba})_{2}(130 \mathrm{mg}, 226 \mu \mathrm{mol}),(R)$-segphos (155 mg, $\left.254 \mu \mathrm{mol}\right)$, and $2 \mathbf{a}(460 \mathrm{mg}, 2.74 \mathrm{mmol})$ was dissolved in THF $(15 \mathrm{~mL})$. After stirring the solution at $40{ }^{\circ} \mathrm{C}$ for $15 \mathrm{~min}$, to this was added 1 (500 mg, $2.28 \mathrm{mmol}$ ) by means of syringe. The mixture was stirred at $40{ }^{\circ} \mathrm{C}$ for $24 \mathrm{~h}$, then filtered through a short pad of $\mathrm{SiO}_{2}$ to remove precipitated inorganic salts. The silica gel pad was washed with a small amount of $\mathrm{Et}_{2} \mathrm{O}$ three times and the combined solution was evaporated to dryness under reduced pressure. The yellow residue was chromatographed on silica gel (hexane/Et $\left.{ }_{2} \mathrm{O}=4 / 1\right)$ to give $3 \mathbf{a}$ (367 mg, 57\% yield) as colorless oil. 
The enantiopurity of $(R)-\mathbf{3 a}$ and $(R)-\mathbf{3 b}$ was determined to be 79 and $87 \%$ ee, respectively, by HPLC analysis with a chiral stationary phase column (3a, Daicel Chiralpak AD, hexane $/ \mathrm{PrOH}=$ 100/1, $0.5 \mathrm{~mL} / \mathrm{min}$; 3b, Daicel Chiralcel OG, hexane $/{ }^{i} \mathrm{PrOH}=98 / 2,0.2 \mathrm{~mL} / \mathrm{min}$ ). The absolute configurations of both $\mathbf{3 a}$ and $\mathbf{3 b}$ were deduced to be $(R)$ by the Lowe-Brewster rule ${ }^{9}$ from the signs of optical rotation $\left(\mathbf{3 a},[\alpha]^{20} \mathrm{D}=-60\left(c 0.99, \mathrm{CHCl}_{3}\right) ; \mathbf{3 b},[\alpha]^{20} \mathrm{D}=-60\left(c 1.01, \mathrm{CHCl}_{3}\right)\right)$.

Dimethyl 2-methyl-2-[5-(trimethylsilyl)penta-2,3-dienyl]propane-1,3-dioate (3a): ${ }^{1} \mathrm{H}$ NMR $\left(500 \mathrm{MHz} \mathrm{CDCl}_{3}\right): \delta 0.01(\mathrm{~s}, 9 \mathrm{H}), 1.25$ (ddd, $J=13.8,8.5$, and $2.3 \mathrm{~Hz}, 1 \mathrm{H}$ ), 1.28 (ddd, $J=13.8,8.8$, and $2.6 \mathrm{~Hz}, 1 \mathrm{H}), 1.43(\mathrm{~s}, 3 \mathrm{H}), 2.52(\mathrm{ddd}, J=13.9,7.8$, and $2.2 \mathrm{~Hz}$, 1H), 2.55 (ddd, $J=13.9,7.8$, and $2.4 \mathrm{~Hz}, 1 \mathrm{H}), 3.710$ (s, 3H), 3.711 (s, 3H), 4.87-4.92 (m, $1 \mathrm{H}), 5.01-5.07(\mathrm{~m}, 1 \mathrm{H}) .{ }^{13} \mathrm{C}\left\{{ }^{1} \mathrm{H}\right\} \mathrm{NMR}\left(125 \mathrm{MHz}, \mathrm{CDCl}_{3}\right): \delta-1.94,17.88,19.76,36.28$, 52.48, 52.53, 53.93, 84.41, 87.24, 172.31, 172.40, 206.37. Anal. Calcd for $\mathrm{C}_{14} \mathrm{H}_{24} \mathrm{O}_{4} \mathrm{Si}$ : C, 59.12; H, 8.50. Found: C, 59.01; H, 8.54.

Diethyl 2-acetylamino-2-[5-(trimethylsilyl)penta-2,3-dienyl]propane-1,3dioate (3b): ${ }^{1} \mathrm{H} \mathrm{NMR}\left(\mathrm{CDCl}_{3}\right): \delta 0.01(\mathrm{~s}, 9 \mathrm{H}), 1.25-1.27(\mathrm{~m}, 8 \mathrm{H}), 2.04(\mathrm{~s}, 3 \mathrm{H}), 2.97(\mathrm{ddd}, J=$ $14.4,8.1$, and $2.2 \mathrm{~Hz}, 1 \mathrm{H}), 3.02$ (ddd, $J=14.4,7.1$, and $2.2 \mathrm{~Hz}, 1 \mathrm{H}), 4.19-4.29(\mathrm{~m}, 4 \mathrm{H}), 4.78$ $4.84(\mathrm{~m}, 1 \mathrm{H}), 5.00-5.06(\mathrm{~m}, 1 \mathrm{H}), 6.78(\mathrm{br}, 1 \mathrm{H}) .{ }^{13} \mathrm{C}\left\{{ }^{1} \mathrm{H}\right\} \mathrm{NMR}\left(\mathrm{CDCl}_{3}\right): \delta-1.95,14.01$, $14.02,17.80,23.03,32.99,62.52$, 62.54, 66.50, 83.22, 87.48, 167.65, 167.76, 168.84, 206.41. Anal. Calcd for $\mathrm{C}_{17} \mathrm{H}_{29} \mathrm{O}_{5} \mathrm{NSi}$ : C, 57.44; H, 8.22; N, 3.94. Found: C, 57.31; H, 8.09; $\mathrm{N}, 4.00$.

Di-tert-butyl $\boldsymbol{N}$-[5-(trimethylsilyl)penta-2,3-dienyl]imino-dicarboxylate (3c): ${ }^{1} \mathrm{H}$ NMR $\left(500 \mathrm{MHz}, \mathrm{CDCl}_{3}\right): \delta 0.01(\mathrm{~s}, 9 \mathrm{H}), 1.28(\mathrm{ddd}, J=13.9,8.3$, and $2.4 \mathrm{~Hz}, 1 \mathrm{H}), 1.32$ (ddd, $J=13.9,8.8$, and $2.7 \mathrm{~Hz}, 1 \mathrm{H}), 1.49$ (s, 18H), 4.10 (ddd, $J=14.9,6.3$, and $2.4 \mathrm{~Hz}, 1 \mathrm{H}$ ), 4.15 (ddd, $J=14.9,5.4$, and $2.7 \mathrm{~Hz}, 1 \mathrm{H}), 5.08-5.17(\mathrm{~m}, 2 \mathrm{H}) .{ }^{13} \mathrm{C}\left\{{ }^{1} \mathrm{H}\right\}$ NMR $(125 \mathrm{MHz}$, $\left.\mathrm{CDCl}_{3}\right): \delta-1.93,17.89,28.11,45.61,82.14,87.45,89.28,152.26,204.42$. Anal. Calcd for $\mathrm{C}_{18} \mathrm{H}_{33} \mathrm{O}_{4} \mathrm{NSi}$ : C, 60.81; H, 9.36; N, 3.94. Found: C, 60.69; H, 9.35; N, 3.88.

$\mathbf{T i C l}_{4}$-Promoted $\mathbf{S}_{\mathbf{E}}{ }^{\prime}$ Reaction of (Allenylmethyl)silanes with Acetals/Aldehyde. The reaction conditions and results are summarized in Table 2. A typical procedure is given for the preparation of $(E)$-dimethyl 2-[3-(1-methoxy-2-phenylethyl)penta-2,4dienyl]-2-methylpropane-1,3-dioate (5ax): Under a nitrogen atmosphere, a solution of $\mathrm{PhCH}_{2} \mathrm{CH}(\mathrm{OMe})_{2}(189 \mathrm{mg}, 1.14 \mathrm{mmol})$ and $\mathrm{TiCl}_{4}\left(1.0 \mathrm{M} \mathrm{CH}_{2} \mathrm{Cl}_{2}\right.$ solution, $\left.1.1 \mathrm{~mL}, 1.1 \mathrm{mmol}\right)$ in $\mathrm{CH}_{2} \mathrm{Cl}_{2}(5 \mathrm{~mL})$ was cooled to $-78{ }^{\circ} \mathrm{C}$ and to this was added (allenylmethyl)silane $(R)-3 \mathbf{a}$ (202 mg, $710 \mu \mathrm{mol} ; 79 \%$ ee) by means of syringe. The solution was stirred at this temperature until total consumption of $\mathbf{3 a}$ (ca. $8 \mathrm{~h}$, checked by TLC). The reaction mixture was quenched with water and extracted with ether twice. The combined organic solution was washed with saturated $\mathrm{NaCl}$ solution, and dried over anhydrous $\mathrm{MgSO}_{4}$. After removal of the solvent, the crude material was chromatographed on silica gel (hexane/ $\left.\mathrm{Et}_{2} \mathrm{O}=4 / 1\right)$ to give the conjugated diene $(219 \mathrm{mg}$, $89 \%$ yield; 5ax) as colorless oil. The characterization data of the 1,3-dienyl products are listed below.

The enantiopurity of the products (see Table 2) were determined by HPLC analysis with a chiral stationary phase column Daicel Chiralcel OD-H (5ax, hexane/ $i \mathrm{PrOH}=100 / 1,0.2 \mathrm{~mL} / \mathrm{min}$; 5ay, double columns connected in series, hexane $/ \mathrm{PrOH}=500 / 1,0.5 \mathrm{~mL} / \mathrm{min} ; \mathbf{5 b x}$, hexane $/ i \mathrm{PrOH}$ $=98 / 2,0.5 \mathrm{~mL} / \mathrm{min} ; \mathbf{5 b y}$, hexane $\left./{ }^{i} \mathrm{PrOH}=500 / 1,0.5 \mathrm{~mL} / \mathrm{min}\right)$.

The absolute configurations of both $\mathbf{5 a y}$ and $\mathbf{5 b y}$ were determined as follows. The diene $\mathbf{5}$ was treated with a mixture of $\mathrm{K}_{2} \mathrm{CO}_{3} / \mathrm{NaIO}_{4} / \mathrm{KMnO}_{4}$ (6 equiv/16 equiv/2.3 equiv) in ${ }^{t} \mathrm{BuOH} / \mathrm{H}_{2} \mathrm{O}$ 
$(1 / 2 \mathrm{v} / \mathrm{v})$ for $12 \mathrm{~h}$. By this treatment, both 5 ay and $\mathbf{5 b y}$ were converted into ${ }^{t} \mathrm{BuCH}(\mathrm{OMe}) \mathrm{COOH}$. The crude carboxylic acid was treated with etherial $\mathrm{CH}_{2} \mathrm{~N}_{2}$, and then the obtained methyl ester was purified by vacuum transfer. The absolute configuration of the ester from both 5ay and $\mathbf{5 b y}$, ${ }^{t} \mathrm{BuCH}(\mathrm{OMe}) \mathrm{COOMe}$, was determined to be $(S)$ by comparison of retention time on the chiral HPLC with that of an authentic sample $(S)-t_{\mathrm{BuCH}}(\mathrm{OMe}) \mathrm{COOMe}$, which was prepared from commercially available $(S)-{ }^{t} \mathrm{BuCH}(\mathrm{OH}) \mathrm{COOH}$ (Aldrich). The methyl ester ${ }^{t} \mathrm{BuCH}(\mathrm{OMe}) \mathrm{COOMe}$ was too volatile to be purified in a small scale, and thus determination of the absolute configuration by the signs of optical rotation was given up.

(E)-Dimethyl 2-[3-(1-methoxy-2-phenylethyl)penta-2,4-dienyl]-2methylpropane-1,3-dioate (5ax): ${ }^{1} \mathrm{H}$ NMR $\left(\mathrm{CDCl}_{3}\right): \delta 1.33(\mathrm{~s}, 3 \mathrm{H}), 2.75(\mathrm{dd}, J=14.9$ and $8.1 \mathrm{~Hz}, 1 \mathrm{H}), 2.78(\mathrm{dd}, J=14.9$ and $7.8 \mathrm{~Hz}, 1 \mathrm{H}), 2.84(\mathrm{dd}, J=14.2$ and $7.3 \mathrm{~Hz}, 1 \mathrm{H}), 2.88$ (dd, $J$ $=14.2$ and $5.4 \mathrm{~Hz}, 1 \mathrm{H}), 3.17(\mathrm{~s}, 3 \mathrm{H}), 3.69(\mathrm{~s}, 3 \mathrm{H}), 3.70(\mathrm{~s}, 3 \mathrm{H}), 4.02(\mathrm{dd}, J=7.3$ and $5.4 \mathrm{~Hz}$, $1 \mathrm{H}), 5.23(\mathrm{~d}, J=11.5 \mathrm{~Hz}, 1 \mathrm{H}), 5.40(\mathrm{dd}, J=8.1$ and $7.8 \mathrm{~Hz}, 1 \mathrm{H}), 5.46(\mathrm{~d}, J=17.8 \mathrm{~Hz}, 1 \mathrm{H})$, $6.57(\mathrm{dd}, J=17.8$ and $11.5 \mathrm{~Hz}, 1 \mathrm{H}), 7.17-7.19(\mathrm{~m}, 3 \mathrm{H}), 7.25-7.28(\mathrm{~m}, 2 \mathrm{H}) .{ }^{13} \mathrm{C}\left\{{ }^{1} \mathrm{H}\right\} \mathrm{NMR}$ $\left(\mathrm{CDCl}_{3}\right): \delta 19.81,33.36,42.13,52.55,52.56,53.71,56.34,84.46,116.19,124.53,126.06$, 128.11, 129.16, 130.89, 138.89, 139.22, 172.19, 172.25. Anal. Calcd for $\mathrm{C}_{20} \mathrm{H}_{26} \mathrm{O}_{5}$ : C, 69.34; H, 7.56. Found: C, 69.45; H, 7.64.

(E)-Dimethyl 2-[3-(1-methoxy-2,2-dimethylpropyl)penta-2,4-dienyl]-2methylpropane-1,3-dioate (5ay): ${ }^{1} \mathrm{H} \mathrm{NMR}\left(\mathrm{CDCl}_{3}\right): \delta 0.85(\mathrm{~s}, 9 \mathrm{H}), 1.44(\mathrm{~s}, 3 \mathrm{H}), 2.81(\mathrm{dd}, J$ $=14.8$ and $7.6 \mathrm{~Hz}, 1 \mathrm{H}), 2.90(\mathrm{dd}, J=14.8$ and $8.2 \mathrm{~Hz}, 1 \mathrm{H}), 3.16(\mathrm{~s}, 3 \mathrm{H}), 3.56(\mathrm{~s}, 1 \mathrm{H}), 3.72(\mathrm{~s}$, $6 \mathrm{H}), 5.16(\mathrm{~d}, J=11.5 \mathrm{~Hz}, 1 \mathrm{H}), 5.29(\mathrm{~d}, J=17.7 \mathrm{~Hz}, 1 \mathrm{H}), 5.42(\mathrm{dd}, J=8.2$ and $7.6 \mathrm{~Hz}, 1 \mathrm{H})$, $6.61(\mathrm{dd}, J=17.7$ and $11.5 \mathrm{~Hz}, 1 \mathrm{H}) .{ }^{13} \mathrm{C}\left\{{ }^{1} \mathrm{H}\right\} \mathrm{NMR}\left(\mathrm{CDCl}_{3}\right): \delta 20.08,26.76,33.72,35.66$, 52.58 (2C), 53.89, 56.71, 88.79, 115.72, 125.07, 133.94, 138.10, 172.37, 172.39. Anal. Calcd for $\mathrm{C}_{17} \mathrm{H}_{28} \mathrm{O}_{5}$ : C, 65.36; $\mathrm{H}, 9.03$. Found: $\mathrm{C}, 65.07 ; \mathrm{H}, 9.01$.

(E)-Dimethyl 2-[3-(1-hydroxy-2-phenylethyl)penta-2,4-dienyl]-2methylpropane-1,3-dioate (5az): ${ }^{1} \mathrm{H}$ NMR $\left(\mathrm{CDCl}_{3}\right): \delta 1.36(\mathrm{~s}, 3 \mathrm{H}), 1.71(\mathrm{br}, 1 \mathrm{H}), 2.71$ (dd, $J=13.9$ and $8.7 \mathrm{~Hz}, 1 \mathrm{H}), 2.75(\mathrm{dd}, J=14.8$ and $7.7 \mathrm{~Hz}, 1 \mathrm{H}), 2.81(\mathrm{dd}, J=14.8$ and $8.1 \mathrm{~Hz}$, $1 \mathrm{H}), 3.00(\mathrm{dd}, J=13.9$ and $3.8 \mathrm{~Hz}, 1 \mathrm{H}), 3.70(\mathrm{~s}, 3 \mathrm{H}), 3.71(\mathrm{~s}, 3 \mathrm{H}), 4.62(\mathrm{dd}, J=8.7$ and 3.8 $\mathrm{Hz}, 1 \mathrm{H}), 5.26(\mathrm{dt}, J=11.5$ and $1.2 \mathrm{~Hz}, 1 \mathrm{H}), 5.41(\mathrm{~d}, J=17.8 \mathrm{~Hz}, 1 \mathrm{H}), 5.59(\mathrm{dd}, J=8.1$ and 7.7 $\mathrm{Hz}, 1 \mathrm{H}), 6.61$ (ddt, $J=17.8,11.5$, and $1.2 \mathrm{~Hz}, 1 \mathrm{H}), 7.21-7.24(\mathrm{~m}, 3 \mathrm{H}), 7.29-7.32(\mathrm{~m}, 2 \mathrm{H})$. ${ }^{13} \mathrm{C}\left\{{ }^{1} \mathrm{H}\right\} \mathrm{NMR}\left(\mathrm{CDCl}_{3}\right): \delta 19.87,33.27,43.48,52.57,52.60,53.79,73.11,115.75,123.07$, $126.51,128.47,129.34,130.98,138.63,142.01,172.25,172.31$. Anal. Calcd for $\mathrm{C}_{19} \mathrm{H}_{24} \mathrm{O}_{5}$ : C, 68.66; H, 7.28. Found: C, 68.61; H, 7.33.

(E)-Diethyl 2-acetylamino-2-[3-(1-methoxy-2-phenylethyl)penta-2,4dienyl]propane-1,3-dioate $(\mathbf{5 b x}):{ }^{1} \mathrm{H}$ NMR $\left(\mathrm{CDCl}_{3}\right): \delta 1.24(\mathrm{t}, J=7.1 \mathrm{~Hz}, 3 \mathrm{H}), 1.25(\mathrm{t}, J=$ $7.2 \mathrm{~Hz}, 3 \mathrm{H}), 1.97(\mathrm{~s}, 3 \mathrm{H}), 2.81(\mathrm{dd}, J=14.2$ and $7.8 \mathrm{~Hz}, 1 \mathrm{H}), 2.86(\mathrm{dd}, J=14.2$ and $4.9 \mathrm{~Hz}$, $1 \mathrm{H}), 3.15(\mathrm{~s}, 3 \mathrm{H}), 3.24(\mathrm{~d}, J=8.1 \mathrm{~Hz}, 2 \mathrm{H}), 4.05(\mathrm{dd}, J=7.8$ and $4.9 \mathrm{~Hz}, 1 \mathrm{H}), 4.10-4.27(\mathrm{~m}$, $4 \mathrm{H}), 5.20(\mathrm{~d}, J=11.5 \mathrm{~Hz}, 1 \mathrm{H}), 5.33(\mathrm{t}, J=8.1 \mathrm{~Hz}, 1 \mathrm{H}), 5.44(\mathrm{~d}, J=17.8 \mathrm{~Hz}, 1 \mathrm{H}), 6.55(\mathrm{dd}, J$ $=17.8$ and $11.5 \mathrm{~Hz}, 1 \mathrm{H}), 6.66(\mathrm{br}, 1 \mathrm{H}), 7.18-7.22(\mathrm{~m}, 3 \mathrm{H}), 7.26-7.29(\mathrm{~m}, 2 \mathrm{H}) .{ }^{13} \mathrm{C}\left\{{ }^{1} \mathrm{H}\right\} \mathrm{NMR}$ $\left(\mathrm{CDCl}_{3}\right): \delta 13.94,13.95,22.98,30.36,42.31,56.40,62.61,62.64,66.11,83.72,115.87$, $122.81,126.12,128.12,129.13,130.92,139.15,139.90,167.54,167.56,169.03$. Anal. Calcd for $\mathrm{C}_{23} \mathrm{H}_{31} \mathrm{O}_{6} \mathrm{~N}$ : C, 66.17; H, 7.48; N, 3.35. Found: C, 65.90; H, 7.50; N, 3.27. 
(E)-Diethyl 2-acetylamino-2-[3-(1-methoxy-2,2-dimethylpropyl)penta-2,4dienyl]propane-1,3-dioate (5by): ${ }^{1} \mathrm{H}$ NMR $\left(\mathrm{CDCl}_{3}\right): \delta 0.84(\mathrm{~s}, 9 \mathrm{H}), 1.26(\mathrm{t}, J=7.3 \mathrm{~Hz}$, $6 \mathrm{H}), 2.01(\mathrm{~s}, 3 \mathrm{H}), 3.14(\mathrm{~s}, 3 \mathrm{H}), 3.28(\mathrm{dd}, J=15.1$ and $7.8 \mathrm{~Hz}, 1 \mathrm{H}), 3.37(\mathrm{dd}, J=15.1$ and 8.3 $\mathrm{Hz}, 1 \mathrm{H}), 3.59(\mathrm{~s}, 1 \mathrm{H}), 4.19-4.28(\mathrm{~m}, 4 \mathrm{H}), 5.14(\mathrm{~d}, J=11.5 \mathrm{~Hz}, 1 \mathrm{H}), 5.29(\mathrm{~d}, J=17.6 \mathrm{~Hz}, 1 \mathrm{H})$, $5.31(\mathrm{dd}, J=8.3$ and $7.8 \mathrm{~Hz}, 1 \mathrm{H}), 6.61(\mathrm{dd}, J=17.6$ and $11.5 \mathrm{~Hz}, 1 \mathrm{H}), 6.75(\mathrm{br}, 1 \mathrm{H}) .{ }^{13} \mathrm{C}\left\{{ }^{1} \mathrm{H}\right\}$ NMR ( $\left.\mathrm{CDCl}_{3}\right): \delta 13.97(2 \mathrm{C}), 23.03,26.69,30.61,35.57,56.70,62.64,62.66,66.23,88.14$, 115.56, 123.47, 133.82, 138.93, 167.73, 167.75, 169.05. Anal. Calcd for $\mathrm{C}_{20} \mathrm{H}_{33} \mathrm{O}_{6} \mathrm{~N}$ : C, 62.64; H, 8.67; N, 3.65. Found: C, 62.43; H, 8.95; N, 3.37.

\section{References}

(1) Roux, M.; Santelli, M.; Parrain, J.-L. Org. Lett. 2000, 2, 1701.

(2) Tatsuno, A.; Yoshida, T.; Otsuka, S. Inorg. Synth. 1979, 19, 220.

(3) Ogasawara, M.; Yoshida, K.; Hayashi, T. Organometallics 2000, 19, 1567.

(4) Clerici, A.; Pastori, N.; Porta, O. Tetrahedron 1998, 54, 15679.

(5) Ukai, T.; Kawazura, H.; Ishii, Y.; Bonnet, J. J.; Ibers, J. A. J. Organomet. Chem. 1974, $65,253$.

(6) Saito, T.; Yokozawa, T.; Ishizaki, T.; Moroi, T.; Sayo, N.; Miura, T.; Kumobayashi, H. Adv. Synth. Catal. 2001, 343, 264.

(7) Ogasawara, M.; Ikeda, H.; Hayashi, T. Angew. Chem., Int. Ed. 2000, 39, 1042.

(8) Ogasawara, M.; Ikeda, H.; Nagano, T.; Hayashi, T. J. Am. Chem. Soc. 2001, 123, 2089.

(9) (a) Lowe, G. Chem. Commun. 1965, 411. (b) Brewster, J. H. Topics in Stereochemistry 1967, 2,1 . 